\title{
Analysis of the stromal cell-derived factor $1-3$ 'A gene polymorphism in pancreatic cancer
}

\author{
GEORGE E. THEODOROPOULOS ${ }^{1}$, GEORGE S. PANOUSSOPOULOS ${ }^{1}$, \\ NIKOLAOS V. MICHALOPOULOS ${ }^{1}$, CONSTANTINOS P. ZAMBIRINIS ${ }^{1}$, STILIANI TAKA ${ }^{2}$, \\ PARASKEVAS STAMOPOULOS ${ }^{1}$, MARIA GAZOULI ${ }^{2}$ and G. ZOGRAFOS ${ }^{1}$ \\ ${ }^{1}$ First Propaedeutic Department of Surgery, Hippocration University Hospital; \\ ${ }^{2}$ Department of Biology, School of Medicine, University of Athens, Athens, Greece
}

Received January 12, 2010; Accepted May 6, 2010

DOI: $10.3892 / \mathrm{mmr} 00000319$

\begin{abstract}
Stromal cell derived factor-1 (SDF-1), a CXC chemokine that plays an important role in the tumor growth, angiogenesis and metastasis of tumor cells, has a polymorphism at position 801 of its 3 -untranslated region, known as SDF1-3'A. The SDF1-3'A polymorphism has been investigated in various types of cancer, but no information is currently available on its role in pancreatic cancer. In this study, 80 pancreatic cancer patients and 160 normal healthy control subjects were investigated for the genotype and allelic frequencies of the SDF-1 gene using PCR-RFLP. The genotype frequencies for GG, GA and AA were 21.25, 77.5 and $1.25 \%$ in patients, and $42.5,55$ and $2.5 \%$ in healthy subjects, respectively. The A carrier group (GA+AA genotype) and the $A$ allele were overrepresented among the patients with pancreatic cancer ( $\mathrm{p}=0.015$ and $\mathrm{p}=0.031$, respectively). The GA+AA genotype was statistically correlated with an advanced $\mathrm{T}$ stage and the presence of lymph node metastasis, and displayed a clear trend towards significance in relation to the presence of distant metastatic disease $(\mathrm{p}=0.061)$. Only $\mathrm{T}$ stage was significantly related to A allele frequency $(\mathrm{p}=0.004)$. SDF1-3' A allele carriers were more prevalent among cancer patients than among normal subjects. SDF1-3' A allele carrier status may imply a higher risk of pancreatic cancer, while the presence of the A allele in pancreatic cancer patients may be related to aggressive features of this malignancy.
\end{abstract}

\section{Introduction}

Pancreatic cancer remains one of the most lethal human cancers with an exceedingly poor prognosis due to the late occurrence of symptoms and its high metastatic potential (1).

Correspondence to: Dr Nikolaos V. Michalopoulos,

9 Alexandroupoleos Str., Athens 115 27, Greece

E-mail:nmichal@med.uoa.gr

Key words: pancreatic cancer, stromal cell derived factor-1, CXCL12, polymorphisms
The aggressive behavior and lack of sensitivity of pancreatic cancer to most treatment options render this tumor a major cause of cancer-related death, with a mortality rate virtually equal to its incidence (2). In 2002, there were approximately 232,000 cases of occurrence and 226,000 deaths due to the disease worldwide (3). Pancreatic cancer remains multifactorial in its etiology and heterogeneous in its development and disease progression. Enhancing our understanding of the molecular biology of malignant pancreatic disease will aid in bridging the gaps in the comprehension of the pathogenesis of this neoplasm, and may improve the clinical outcome for patients. Although smoking is firmly established as the strongest risk factor for pancreatic cancer, there is also considerable interaction between genetic and environmental factors (4). Several studies have examined the association between genetic polymorphisms of smoking- and diet-related carcinogen detoxifying enzymes and pancreatic cancer (5-7). However, relatively scarce data exist regarding the genetic polymorphisms of factors with documented invasive and metastatic roles in this malignancy.

Chemokines are low-molecular-weight peptide ligands that function as signaling molecules in the trafficking of leukocytes and other motile cells $(8,9)$. They have been classified into four subgroups, $\mathrm{C}, \mathrm{CC}, \mathrm{CXC}$ and $\mathrm{CX} 3 \mathrm{C}$, based on the positioning of four conserved cysteine residues, which form two disulfide bonds (10). The binding of chemokines to their G-proteinlinked receptors on target cells leads to signal transduction events involving the generation of inositol triphosphate, the release of intracellular calcium and the activation of protein kinase $\mathrm{C}$ and proteins of the Ras and Rho families (11). Chemokine receptor activation may lead to growth and adhesion, as well as to directional site-specific cancer cell migration and implantation in distinct metastasis-harboring organs (12). Chemokines orchestrate T-cell migration during immune and inflammatory responses as well as stem cell and progenitor immune cell migration to and from various organs and tissues in hematopoeisis and development $(13,14)$. Among the chemokines is stromal cell-derived factor-1 (SDF-1), also known as CXCL12, which was initially cloned by Tashiro et al. It was later identified as a growth factor for B-cell progenitors, and a chemotactic factor for T-cells and monocytes, and was found in B-cell lymphopoiesis and bonemarrow myelopoiesis 
(15-18). SDF-1 principally binds to the CXCR4 receptor, resulting in a SDF-1/CXCR4 receptor-ligand system involving a one-on-one interaction $(19,20)$. A second newly-discovered receptor, RDC-1/CXCR7, reportedly binds SDF-1, although its functions remain unclear (21).

Recently, CXCR4 has attracted attention as it may play a crucial role in the metastatic processes of many types of tumors, including colorectal, breast and oral squamous-cell carcinoma (22-24). Further data from in vitro and murine in vivo tumor models have underlined the key role of CXCR4 in tumor cell malignancy; the activation of CXCR4 by SDF-1 was shown to induce the migration, invasion and angiogenesis of cancer cells, while inhibition of the SDF-1-CXCR4 axis resulted in reduced metastatic potential $(25,26)$. CXCR4 expression is detected in both pancreatic cancer tissues and cancer cell lines (26-29).

To date, three isoforms of SDF-1 have been described. The beta variant has a guanine to adenine $(\mathrm{G} \rightarrow \mathrm{A})$ single nucleotide polymorphism at position 801 of the 3 '-untranslated region of its gene (30-32). Allele $\mathrm{A}$ is probably a target for Cis-acting factors, capable of up-regulating the expression of SDF-1 protein $(30,32,33)$. It has been suggested that homozygotes for SDF1-3'A (3'A/3'A) produce more SDF-1 than wild-type ones $\left(3^{\prime} \mathrm{G} / 3^{\prime} \mathrm{G}\right)$, an observation which is in need of confirmation $(32,34,35)$. This single nucleotide polymorphism of the SDF-1 gene has been investigated in type 1 diabetes and HIV-1 infection, and has been reported to be associated with an increased risk of non-Hodgkin's lymphoma in HIV-1 infected individuals $(30,34,35)$. The SDF-1 $\mathrm{G} \rightarrow \mathrm{A}$ polymorphism has been investigated in various cancers, but no information is currently available regarding its role in pancreatic cancer (36-44).

We hypothesized that the A allele is associated with increased risk of pancreatic cancer and an aggressive tumor phenotype, and sought to explore the role of different SDF-1 genotypes in the susceptibility to and behavior of this disease.

\section{Materials and methods}

A case-control analysis was conducted on patients with ductal adenocarcinoma admitted to the First Propaedeutic Department of Surgery, Hippocration University Hospital (Athens, Greece). A total of 80 specimens were obtained intra-operatively from patients with pancreatic cancer (mean age \pm SEM 66.85 \pm 1.16 ; median 70; range 27-82) who underwent Whipple surgery or from patients with inoperable or metastatic disease who underwent a biopsy of the primary site or of the liver metastasis as a sole procedure or as part of a palliative 'bypass' surgery for pancreatic cancer. The morphological classification of the carcinomas and the staging of the disease were conducted according to the World Health Organization specifications and the UICC staging system, respectively. An additional 160 age- and gender-matched healthy control individuals (blood donors randomly selected from our DNA database) without evidence of malignancy or autoimmune disease were enrolled. All participants gave their informed consent prior to inclusion in the study.

Genomic DNA was isolated using a NucleoSpin Blood kit (Macherey-Nagel, GmbH \& Co. KG, Germany) according to the manufacturer's instructions. Genotyping analysis was performed using PCR-RFLP with the primers
Table I. Patient and tumor characteristics.

\begin{tabular}{|c|c|}
\hline Characteristic & No. $(\%)$ \\
\hline Total number & 80 \\
\hline Median age (years) & 70 \\
\hline \multicolumn{2}{|l|}{ Gender } \\
\hline Male & 43 \\
\hline Female & 37 \\
\hline \multicolumn{2}{|l|}{ T-status } \\
\hline 1 & $5 \quad(6)$ \\
\hline 2 & $13(17)$ \\
\hline 3 & $53(66)$ \\
\hline 4 & $9(11)$ \\
\hline \multicolumn{2}{|l|}{ N-status } \\
\hline 0 & $38(48)$ \\
\hline+ & $35(44)$ \\
\hline Unknown & $7(8)$ \\
\hline \multicolumn{2}{|l|}{ M-status } \\
\hline 0 & $68(85)$ \\
\hline 1 & $12(15)$ \\
\hline \multicolumn{2}{|l|}{ Grade } \\
\hline 1 & $6 \quad(7)$ \\
\hline 2 & $42(53)$ \\
\hline 3 & $27(34)$ \\
\hline Unknown & $5(6)$ \\
\hline \multicolumn{2}{|c|}{ Perivascular infiltration } \\
\hline Absent & $65(81)$ \\
\hline Present & 15 (19) \\
\hline
\end{tabular}

SDF-F, 5'-CAGTCAACCTGGGCAAAGCC-3' and SDF-R, 5'-CCTGAGAGTCCTTTTGCGGG-3' (GenBank accession number L36033). Amplicons of 293 bp were subjected to restriction digestion by the HpaII enzyme. SDF-1 wild-type alleles yielded 100 and 193 bp products, while SDF-1 3' A alleles (polymorphic) yielded a 293 bp product. The products were analyzed by electrophoresis on a $2 \%$ agarose gel and visualized using UV fluorescence after staining with ethidium bromide.

Statistical calculations were performed using the SPSS for Windows software package (SPSS Inc., Chicago, IL, USA). Frequency and susceptibilities of mutations were compared using the $\chi^{2}$ test. Odds ratios (OR) and 95\% confidence intervals $(95 \% \mathrm{CI})$ were calculated with the corresponding $\chi^{2}$ distribution test. The p-values obtained were two-tailed and determined to be significant at $\mathrm{p}<0.05$. Hardy-Weinberg equilibrium was verified by calculating the expected frequencies and numbers and was tested separately in patients and in controls using the goodness-of-fit $\chi^{2}$ test.

\section{Results}

Tumor characteristics are shown in Table I. The SDF-1 genotype and allele distribution among patients and healthy controls 
Table II. Distribution of SDF-1 genotypes and alleles in patients and controls.

\begin{tabular}{|c|c|c|c|c|}
\hline & Patients (\%) & Controls (\%) & $\mathrm{p}$-value & OR $(95 \% \mathrm{CI})$ \\
\hline \multicolumn{5}{|c|}{ Genotypic frequencies } \\
\hline GG & $17(21.25)$ & $68(42.5)$ & \multirow{3}{*}{0.015} & 1.00 (reference) \\
\hline $\mathrm{AG}+\mathrm{AA}$ & $63(78.75)$ & $92(57.5)$ & & $2.74(1.47-5.09)$ \\
\hline Total & $80(100)$ & $160(100)$ & & \\
\hline \multicolumn{5}{|c|}{ Allelic frequencies } \\
\hline $\mathrm{G}$ & $96(60)$ & $224(72)$ & \multirow{3}{*}{0.031} & 1.00 (reference) \\
\hline A & $64(40)$ & $96(28)$ & & $1.55(1.04-2.31)$ \\
\hline Total & $160(100)$ & $320(100)$ & & \\
\hline
\end{tabular}

is presented in Table II. The observed genotype frequencies were in accordance with Hardy-Weinberg equilibrium. Only 1 patient and 4 cases among the control group presented with the AA genotype; as a result, these were grouped together with the GA cases during the statistical analysis (Table II). The genotype frequencies for GG, GA and AA were 21.25, 77.5 and $1.25 \%$ in patients, and $42.5,55$ and $2.5 \%$ in healthy subjects, respectively. GA+AA genotype frequency and A allele distribution were significantly different between pancreatic cancer cases and controls. The A carriers (GA+AA genotype) and the A allele were overrepresented among the pancreatic cancer cases $(\mathrm{p}=0.015$ and $\mathrm{p}=0.031$, respectively) (Table II).

Concerning tumor characteristics, a statistically significant association was detected between the SDF-1 GA A carrier group (GA+AA genotype) and advanced T stage as well as the presence of lymph node metastasis $(\mathrm{p}=0.0001$ and $\mathrm{p}=0.003)$, while a clear trend towards significance was observed in relation to the presence of distant metastatic disease $(p=0.061)$ (Table III). Only T stage was significantly related to A allele frequency $(\mathrm{p}=0.004)$ (Table III).

\section{Discussion}

The diverse cellular origins of SDF-1 and its constitutive expression in various organs warrant the study of this chemokine as an organ microenvironment 'host' factor in malignant and non-malignant pathologies $(19,45)$. The role of SDF-1 as a significant 'host' factor is outlined by the model for metastasis proposed by Muller et al, according to which specific chemokines, secreted by particular target organs to which breast cancer metastasizes, serve to attract the circulating breast cancer cells that express receptors for these chemokines (46). The authors identified one such chemokine/ receptor pair as SDF-1/CXCR4 (46). Tumor cells, including breast cancers, were found to express high levels of the CXCR4 receptor, whereas human organs targeted by metastatic breast tumor cells expressed high levels of SDF-1 (46). Additional supporting evidence for the role of SDF-1 as a 'host' factor is that SDF-1 is a powerful chemoattractant secreted in the bone marrow, and functions to retain progenitor stem cells within the bone marrow (47).

It is commonly accepted that the clinical behavior of tumors depends on the relationship between tumor cells and the host. The majority of molecular studies have focused on cancer-associated genes or carcinogen-metabolizing genes; relatively few have been carried out to screen for genetic polymorphisms with the aim of uncovering the potential predictive role of such 'host' factors in cancer susceptibility or any of the carcinogenesis elements.

The most compelling evidence for the role of the SDF-1 $\mathrm{G} \rightarrow \mathrm{A}$ polymorphism in malignancy is provided by previous genotype and allele-frequency studies showing that this specific polymorphism is associated with a susceptibility to breast, lung and prostate cancer $(36,38,40-42)$. In the current study, we attempted to determine whether an association exists between this SDF-1 gene variant and pancreatic cancer. The data indicate that, compared with the controls, the genotype distribution and allelic frequencies were significantly associated with pancreatic cancer. Consistent with the aforementioned reports, our study is the first to document the positive association of the A allele and the GA genotype with an increased risk of pancreatic cancer development. On the other hand, current studies on colorectal cancer have failed to suggest a serious impact of the SDF-1 polymorphism on the risk of colorectal cancer development $(43,44)$. Hypothetically, different mechanisms involved in the pathogenesis of colorectal carcinogenesis, along with a host of modifying molecular events, may account for this lack of association, in contrast to the documented role of the SDF-1 polymorphism in breast, lung, prostate and pancreatic cancer (36,38,40-42). In other words, based on tissue origin, the influence of the SDF-1 gene polymorphism may contribute differentially between malignancies in mediating tumor progression, angiogenesis, metastasis and leukocyte migration.

The influence of the SDF-1 GA genotype and the SDF-1 A allele on pancreatic cancer development and progression remains to be determined for as long as the functional importance of the A allele in the expression of SDF-1 transcript and protein is largely obscured. As demonstrated in a study focusing on colorectal cancer, little is known about the interactions of various cancer-related genetic pathways and SDF-1/ CXCL12-CXCR4 signaling (43). The association of SDF-1/ CXCL12 with CXCR4 activates the receptor, which leads to the activation of multiple signaling pathways and in turn regulates locomotion, chemotaxis, adhesion and the secretion of tumor CXCR4-positive cells. Additionally, the results indicate a strong association between the GA genotype and 
Table III. Tumor characteristics in relation to SDF-1 genotypes and alleles*.

\begin{tabular}{|c|c|c|c|c|}
\hline \multirow[t]{2}{*}{ Characteristic } & \multicolumn{2}{|c|}{ Genotype $(\mathrm{n}=80)$} & \multicolumn{2}{|c|}{ Allele $(n=160)$} \\
\hline & $\mathrm{GG}(\%)$ & GA or AA $(\%)$ & $\mathrm{G}(\%)$ & $\mathrm{A}(\%)$ \\
\hline \multicolumn{5}{|l|}{ T status } \\
\hline $\mathrm{T} 1,2$ & $11(14)$ & 7 (9) & $29(18)$ & $7 \quad(4)$ \\
\hline \multirow[t]{2}{*}{$\mathrm{T} 3,4$} & $6(7)$ & $56(70)$ & $67(42)$ & $57(36)$ \\
\hline & \multicolumn{2}{|c|}{$\mathrm{p}=0.0001$} & \multicolumn{2}{|c|}{$\mathrm{p}=0.004$} \\
\hline \multicolumn{5}{|l|}{$\mathrm{N}$ status $(\mathrm{n}=73)$} \\
\hline N0 & 13 (18) & $25(34)$ & $50(34)$ & $26(18)$ \\
\hline \multirow[t]{2}{*}{$\mathrm{N}+$} & $2(3)$ & $33(45)$ & $37(25)$ & $33(23)$ \\
\hline & \multicolumn{2}{|c|}{$\mathrm{p}=0.003$} & \multicolumn{2}{|c|}{$\mathrm{p}=0.112$} \\
\hline \multicolumn{5}{|l|}{ M status } \\
\hline M0 & $17(21)$ & $51(64)$ & $85(53)$ & $51(32)$ \\
\hline \multirow[t]{2}{*}{ M1 } & $0 \quad(0)$ & $12(15)$ & 11 & $13(8)$ \\
\hline & \multicolumn{2}{|c|}{$\mathrm{p}=0.061$} & \multicolumn{2}{|c|}{$\mathrm{p}=0.124$} \\
\hline \multicolumn{5}{|l|}{ Stage } \\
\hline Stage I,II & $14(18)$ & $52(65)$ & $80(50)$ & $50(31)$ \\
\hline \multirow[t]{2}{*}{ Stage III,IV } & $3(4)$ & $11(13)$ & $16(10)$ & $14(9)$ \\
\hline & \multicolumn{2}{|c|}{$\mathrm{p}=0.506$} & \multicolumn{2}{|c|}{$\mathrm{p}=0.408$} \\
\hline \multicolumn{5}{|l|}{ Grade $(n=75)$} \\
\hline 1,2 & $10(13)$ & $38(51)$ & $58(39)$ & $38(25)$ \\
\hline \multirow[t]{2}{*}{3} & $6(8)$ & $21(28)$ & $32(21)$ & $22(15)$ \\
\hline & \multicolumn{2}{|c|}{$\mathrm{p}=0.554$} & \multicolumn{2}{|c|}{$\mathrm{p}=0.513$} \\
\hline \multicolumn{5}{|c|}{ Perivascular infiltration } \\
\hline Absent & $17(21)$ & $48(60)$ & $81(51)$ & $49(31)$ \\
\hline \multirow[t]{2}{*}{ Present } & $0 \quad(0)$ & $15(19)$ & $15(9)$ & $15(9)$ \\
\hline & \multicolumn{2}{|c|}{$\mathrm{p}=0.032$} & \multicolumn{2}{|c|}{$\mathrm{p}=0.215$} \\
\hline
\end{tabular}

*N-status could not be obtained from 7 patients, whereas grading could not be obtained for 5 patients.

or A allele and adverse tumor pathological characteristics, leading us to speculate that, in the presence of the polymorphism, this activation may be enhanced. The mechanisms behind the relationship between the SDF-1 polymorphism and the clinical behavior of the tumors, observed in many of the single nucleotide polymorphisms studied, has been poorly investigated. SDF-1 gene functional alteration, either towards the activating or the repressing side, taking into consideration the biological role of this molecule in the tumor microenvironment as well as the target organs for metastasis, may serve as a rational explanation. It should be kept in mind that the mode of action of chemokines depends heavily on the local environment, and that secreted SDF-1 is thought to act by creating a gradient for CXCR4-bearing cells (27). Moreover, SDF-1 expression is most intense in typical 'homing organs' such as the lungs, bone marrow, liver and lymph nodes, as compared with other non-homing tissues $(29,48)$. Synergies with differential expression or the action of co-factor molecules may be of paramount importance. Therefore, pathophysiologically, endothelial cells that co-express SDF-1 and VCAM-1 mediate tumor cell to endothelial cell attachment; CXCR4 activation by SDF-1 induces $\beta$-integrin expression and the binding of VCAM-1 to endothelial cells $(49,50)$.

The results of the present study indicating the significant association of the SDF-1 GA genotype with the T and $\mathrm{N}$ stages and of the A allele with the $\mathrm{T}$ stage, as well as the observation that all patients with metastatic tumors were positive for the GA genotype, add to growing in vitro and in vivo evidence linking this important chemokine with an aggressive phenotype of pancreatic cancer (26-29). Our data support the in vitro observations of Mori et al, who described a dosedependent SDF-1 stimulation of migration and invasion in pancreatic cancer cells and thus implicated the SDF-1 polymorphism in the gene's protein product in an enhancing role (26). Corroborating this report, the results of Marchesi et al indicated that SDF-1 (CXCR4) expression conferred increased motility and invasion ability to pancreatic tumor cells, while protecting the cells from serum starvation-induced damage and decreasing the rate of apoptosis (28). The role of SDF-1 on the migratory phenotype of pancreatic cancer via the involvement of a paracrine mechanism in the SDF-1/CXCR4 receptor ligand system is outlined by Koshiba et al (27). 
Since many tumors widely express CXCR4, the receptor for SDF-1, this ligand/receptor pair is an interesting therapeutic target in cancer patients $(26,36,41)$. Indeed, antiCXCR4 agents in various cancers are currently undergoing clinical trials. Whether consideration, in terms of patient selection, should be given to the SDF-1 A carrier remains to be determined (36). In addition to considering host factors such as chemokines and tumor factors as criteria for therapy selection, new clinical trials in oncology may focus on their respective polymorphisms as important modifying factors of prognosis and treatment strategy.

\section{References}

1. Evans DB, Lee JE, Pisters PW, et al: Advances in the diagnosis and treatment of adenocarcinoma of the pancreas. Cancer Treat Res 90: 109-125, 1997.

2. Jemal A, Siegel R, Ward E, Murray T, Xu J and Thun MJ: Cancer statistics, 2007. CA Cancer J Clin 57: 43-66, 2007.

3. Parkin DM, Bray F, Ferlay J and Pisani P: Global cancer statistics, 2002. CA Cancer J Clin 55: 74-108, 2005.

4. Vitone LJ, Greenhalf W, McFaul CD, Ghaneh $\mathrm{P}$ and Neoptolemos JP: The inherited genetics of pancreatic cancer and prospects for secondary screening. Best Pract Res Clin Gastroenterol 20: 253-283, 2006.

5. Bartsch H, Malaveille C, Lowenfels AB, Maisonneuve $P$, Hautefeuille A and Boyle P: Genetic polymorphism of $\mathrm{N}$-acetyltransferases, glutathione S-transferase M1 and NAD(P) $\mathrm{H}$ : quinone oxidoreductase in relation to malignant and benign pancreatic disease risk. The International Pancreatic Disease Study Group. Eur J Cancer Prev 7: 215-223, 1998.

6. Duell EJ, Holly EA, Bracci PM, Liu M, Wiencke JK and Kelsey KT: A population-based, case-control study of polymorphisms in carcinogen-metabolizing genes, smoking, and pancreatic adenocarcinoma risk. J Natl Cancer Inst 94: 297-306, 2002

7. Ockenga J, Vogel A, Teich N, Keim V, Manns MP and Strassburg CP: UDP glucuronosyltransferase (UGT1A7) gene polymorphisms increase the risk of chronic pancreatitis and pancreatic cancer. Gastroenterology 124: 1802-1808, 2003.

8. Mellado M, Rodriguez-Frade JM, Manes S and Martinez AC: Chemokine signaling and functional responses: the role of receptor dimerization and TK pathway activation. Annu Rev Immunol 19: 397-421, 2001.

9. Payne AS and Cornelius LA: The role of chemokines in melanoma tumor growth and metastasis. J Invest Dermatol 118 915-922, 2002

10. Rossi D and Zlotnik A: The biology of chemokines and their receptors. Annu Rev Immunol 18: 217-242, 2000.

11. Luster AD: Chemokines - chemotactic cytokines that mediate inflammation. N Engl J Med 338: 436-445, 1998.

12. Ganju RK, Brubaker SA, Meyer J, et al: The alpha-chemokine, stromal cell-derived factor-1alpha, binds to the transmembrane G-protein-coupled CXCR-4 receptor and activates multiple signal transduction pathways. J Biol Chem 273: 23169-23175, 1998.

13. Murdoch $\mathrm{C}$ and Finn A: Chemokine receptors and their role in inflammation and infectious diseases. Blood 95: 3032-3043, 2000 .

14. Mebius RE: Organogenesis of lymphoid tissues. Nat Rev Immunol 3: 292-303, 2003

15. Tashiro K, Tada H, Heilker R, Shirozu M, Nakano T and Honjo T: Signal sequence trap: a cloning strategy for secreted proteins and type I membrane proteins. Science 261: 600-603, 1993.

16. Nagasawa $\mathrm{T}$, Kikutani $\mathrm{H}$ and Kishimoto $\mathrm{T}$ : Molecular cloning and structure of a pre-B-cell growth-stimulating factor. Proc Natl Acad Sci USA 91: 2305-2309, 1994.

17. Bleul CC, Fuhlbrigge RC, Casasnovas JM, Aiuti A and Springer TA: A highly efficacious lymphocyte chemoattractant, stromal cell-derived factor 1 (SDF-1). J Exp Med 184: 1101-1109, 1996.

18. Nagasawa T, Hirota S, Tachibana K, et al: Defects of B-cell lymphopoiesis and bone-marrow myelopoiesis in mice lacking the CXC chemokine PBSF/SDF-1. Nature 382: 635-638, 1996.

19. Bleul CC, Farzan M, Choe H, et al: The lymphocyte chemoattractant SDF-1 is a ligand for LESTR/fusin and blocks HIV-1 entry. Nature 382: 829-833, 1996.
20. Tachibana $\mathrm{K}$, Hirota S, Iizasa $\mathrm{H}$, et al: The chemokine receptor CXCR4 is essential for vascularization of the gastrointestinal tract. Nature 393: 591-594, 1998.

21. Balabanian K, Lagane B, Infantino S, et al: The chemokine SDF-1/CXCL12 binds to and signals through the orphan receptor RDC1 in T lymphocytes. J Biol Chem 280: 35760-35766, 2005.

22. Schimanski CC, Schwald S, Simiantonaki N, et al: Effect of chemokine receptors CXCR4 and CCR7 on the metastatic behavior of human colorectal cancer. Clin Cancer Res 11: 1743-1750, 2005.

23. Kato M, Kitayama J, Kazama S and Nagawa H: Expression pattern of CXC chemokine receptor-4 is correlated with lymph node metastasis in human invasive ductal carcinoma. Breast Cancer Res 5: R144-R150, 2003.

24. Uchida D, Begum NM, Almofti A, et al: Possible role of stromalcell-derived factor-1/CXCR4 signaling on lymph node metastasis of oral squamous cell carcinoma. Exp Cell Res 290: 289-302, 2003.

25. Chen Y, Stamatoyannopoulos G and Song CZ: Down-regulation of CXCR4 by inducible small interfering RNA inhibits breast cancer cell invasion in vitro. Cancer Res 63: 4801-4804, 2003.

26. Mori T, Doi R, Koizumi M, et al: CXCR4 antagonist inhibits stromal cell-derived factor 1-induced migration and invasion of human pancreatic cancer. Mol Cancer Ther 3: 29-37, 2004.

27. Koshiba T, Hosotani R, Miyamoto $\mathrm{Y}$, et al: Expression of stromal cell-derived factor 1 and CXCR4 ligand receptor system in pancreatic cancer: a possible role for tumor progression. Clin Cancer Res 6: 3530-3535, 2000.

28. Marchesi F, Monti P, Leone BE, et al: Increased survival, proliferation, and migration in metastatic human pancreatic tumor cells expressing functional CXCR4. Cancer Res 64: 8420-8427, 2004.

29. Wehler T, Wolfert F, Schimanski CC, et al: Strong expression of chemokine receptor CXCR4 by pancreatic cancer correlates with advanced disease. Oncol Rep 16: 1159-1164, 2006.

30. Watanabe MA, de Oliveira Cavassin GG, Orellana MD, et al: SDF-1 gene polymorphisms and syncytia induction in Brazilian HIV-1 infected individuals. Microb Pathog 35:31-34, 2003.

31. Dean M, Carrington M and O'Brien SJ: Balanced polymorphism selected by genetic versus infectious human disease. Annu Rev Genomics Hum Genet 3: 263-292, 2002.

32. Voevodin A, Samilchuk E and Dashti S: Frequencies of SDF-1 chemokine, CCR-5, and CCR-2 chemokine receptor gene alleles conferring resistance to human immunodeficiency virus type 1 and AIDS in Kuwaitis. J Med Virol 58: 54-58, 1999.

33. Winkler C, Modi W, Smith MW, et al: Genetic restriction of AIDS pathogenesis by an SDF-1 chemokine gene variant. ALIVE Study, Hemophilia Growth and Development Study (HGDS), Multicenter AIDS Cohort Study (MACS), Multicenter Hemophilia Cohort Study (MHCS), San Francisco City Cohort (SFCC). Science 279: 389-393, 1998.

34. Sei S, O'Neill DP, Stewart SK, et al: Increased level of stromal cell-derived factor-1 mRNA in peripheral blood mononuclear cells from children with AIDS-related lymphoma. Cancer Res 61: 5028-5037, 2001

35. Ide A, Kawasaki E, Abiru N, et al: Stromal-cell derived factor-1 chemokine gene variant is associated with type 1 diabetes age at onset in Japanese population. Hum Immunol 64: 973-978, 2003.

36. Hassan S, Baccarelli A, Salvucci O and Basik M: Plasma stromal cell-derived factor-1: host derived marker predictive of distant metastasis in breast cancer. Clin Cancer Res 14: 446-454, 2008.

37. Khademi B, Razmkhah M, Erfani N, Gharagozloo M and Ghaderi A: SDF-1 and CCR5 genes polymorphism in patients with head and neck cancer. Pathol Oncol Res 14: 45-50, 2008.

38. Hirata H, Hinoda Y, Kikuno N, et al: CXCL12 G801A polymorphism is a risk factor for sporadic prostate cancer susceptibility. Clin Cancer Res 13: 5056-5062, 2007.

39. Nahon P, Sutton A, Rufat P, et al: Chemokine system polymorphisms, survival and hepatocellular carcinoma occurrence in patients with hepatitis C virus-related cirrhosis. World J Gastroenterol 14: 713-719, 2008

40. Razmkhah M, Doroudchi M, Ghayumi SM, Erfani N and Ghaderi A: Stromal cell-derived factor-1 (SDF-1) gene and susceptibility of Iranian patients with lung cancer. Lung Cancer 49: 311-315, 2005.

41. Coelho A, Calcada C, Catarino R, Pinto D, Fonseca G and Medeiros R: CXCL12-3' A polymorphism and lung cancer metastases protection: new perspectives in immunotherapy? Cancer Immunol Immunother 55: 639-643, 2006. 
42. Razmkhah M, Talei AR, Doroudchi M, Khalili-Azad T and Ghaderi A: Stromal cell-derived factor-1 (SDF-1) alleles and susceptibility to breast carcinoma. Cancer Lett 225: 261-266, 2005.

43. Dimberg J, Hugander A, Lofgren $S$ and Wagsater D: Polymorphism and circulating levels of the chemokine CXCL12 in colorectal cancer patients. Int J Mol Med 19: 11-15, 2007.

44. Hidalgo-Pascual M, Galan JJ, Chaves-Conde M, et al: Analysis of CXCL12 3'UTR G>A polymorphism in colorectal cancer. Oncol Rep 18: 1583-1587, 2007.

45. Salvucci O, Yao L, Villalba S, Sajewicz A, Pittaluga S and Tosato G: Regulation of endothelial cell branching morphogenesis by endogenous chemokine stromal-derived factor-1. Blood 99: 2703-2711, 2002.

46. Muller A, Homey B, Soto H, et al. Involvement of chemokine receptors in breast cancer metastasis. Nature 410: 50-56, 2001.
47. Petit I, Szyper-Kravitz M, Nagler A, et al: G-CSF induces stem cell mobilization by decreasing bone marrow SDF-1 and up-regulating CXCR4. Nat Immunol 3: 687-94, 2002

48. Phillips RJ, Burdick MD, Lutz M, Belperio JA, Keane MP and Strieter RM: The stromal derived factor-1/CXCL12-CXC chemokine receptor 4 biological axis in non-small cell lung cancer metastases. Am J Respir Crit Care Med 167: 1676-1686, 2003.

49. Burger M, Glodek A, Hartmann T, et al: Functional expression of CXCR4 (CD184) on small-cell lung cancer cells mediates migration, integrin activation, and adhesion to stromal cells. Oncogene 22: 8093-8101, 2003.

50. Cardones AR, Murakami T and Hwang ST: CXCR4 enhances adhesion of B16 tumor cells to endothelial cells in vitro and in vivo via beta(1) integrin. Cancer Res 63: 6751-6757, 2003. 